\title{
Enhanced Synchrony in Epileptiform Activity? Local versus Distant Phase Synchronization in Generalized Seizures
}

\author{
Luis Garcia Dominguez, ${ }^{1}$ Richard A. Wennberg, ${ }^{3}$ William Gaetz, ${ }^{2}$ Douglas Cheyne, ${ }^{2}$ O. Carter Snead III, ${ }^{1}$ and \\ Jose Luis Perez Velazquez ${ }^{1}$ \\ ${ }^{1}$ Brain and Behaviour Program and Division of Neurology, and 2Neuromagnetic Imaging Laboratory, The Hospital for Sick Children, Toronto, Ontario, \\ Canada M5G 1X8, and ${ }^{3}$ Krembil Neuroscience Centre, Toronto Western Hospital, Department of Paediatrics and Institute of Medical Science, University of \\ Toronto, Toronto, Ontario, Canada M5T 2S8
}

Synchronization is a fundamental characteristic of complex systems and a basic mechanism of self-organization. A traditional, accepted perspective on epileptiform activity holds that hypersynchrony covering large brain regions is a hallmark of generalized seizures. However, a few recent reports have described substantial fluctuations in synchrony before and during ictal events, thus raising questions as to the widespread synchronization notion. In this study, we used magnetoencephalographic recordings from epileptic patients with generalized seizures and normal control subjects to address the extent of the phase synchronization (phase locking) in local (neighboring) and distant cortical areas and to explore the ongoing temporal dynamics for particular ranges of frequencies at which synchrony occurs, during interictal and ictal activity. Synchronization patterns were found to differ somewhat depending on the epileptic syndrome, with primary generalized absence seizures displaying more long-range synchrony in all frequency bands studied (3-55 Hz) than generalized tonic motor seizures of secondary (symptomatic) generalized epilepsy or frontal lobe epilepsy. However, all seizures were characterized by enhanced local synchrony compared with distant synchrony. There were fluctuations in the synchrony between specific cortical areas that varied from seizure to seizure in the same patient, but in most of the seizures studied, regardless of semiology, there was a constant pattern in the dynamics of synchronization, indicating that seizures proceed by a recruitment of neighboring neuronal networks. Together, these data indicate that the concept of widespread "hypersynchronous" activity during generalized seizures may be misleading and valid only for very specific neuronal ensembles and circumstances.

Key words: synchrony; phase locking; epilepsy; neocortex; magnetoencephalography; seizures

\section{Introduction}

The correlation or synchronization of cellular activity in the brain is considered to be important for information processing. Determination of neuronal synchronization has been performed using several approaches, from classical cross-correlation to more modern phase synchronization or dynamical interdependence analyses (Schiff et al., 1996; Le Van Quyen et al., 1998; Varela et al., 2001). Although there is evidence that cognitive functions may be mediated by specific patterns of neuronal activity, characterized by rapid changes in synchronization (Friston, 2001; Varela et al., 2001; Lutz et al., 2002), abnormally high levels of neuronal synchrony in extensive brain regions are commonly accepted as the hallmark of epileptiform activity, both ictal (seizures) and interictal. This view is derived from the seemingly highly correlated patterns of activity observed in electroencepha-

Received March 17, 2005; revised July 20, 2005; accepted July 22, 2005.

This work was supported in part by a Discovery grant from the National Science and Engineering Research Council of Canada. We thank Roy Sharma, Stephanie Holowka, and Jason Belkas for technical help and Dr. Steven J. Schifffor critical reading of this manuscript.

Correspondence should be addressed to Dr. Jose Luis Perez Velazquez, Department of Neurology, The Hospital for Sick Children, Room 6535 Hill Wing, 555 University Avenue, Toronto, Ontario, Canada M5G 1X8. E-mail: jose-luis.perez-velazquez@sickkids.ca.

DOI:10.1523/JNEUROSCI.1046-05.2005

Copyright $\odot 2005$ Society for Neuroscience $\quad$ 0270-6474/05/258077-08\$15.00/0 lographic (EEG) recordings during seizures, covering large portions of the neocortex. However, this view is currently being challenged. Although visual interpretation of the EEG recording of a generalized seizure suggests a highly synchronized pattern, more careful analysis of the ictal electroencephalogram reveals that the synchrony between periodic components of a seizure is not nearly perfect. The study by Quiroga et al. (2002) portrays these considerations, with examples of apparently highly synchronized seizure activity that reveal, after a more sophisticated analysis, that the synchrony is no higher than during normal brain activity. Subtle phase shifts diminish the apparent "hypersynchrony" during seizure activity, subtle effects that are only revealed by a more sophisticated analysis.

The physical concept of synchrony, entrainment with phase locking (Hoppensteadt and Izhikevich, 1997), has to be relaxed when applied to noisy brain recordings. For biological time series, neuroscientists use a number of tools that explore the relative information that is contained in one series with respect to another series. In this line of work, concepts such as generalized synchronization (Pikovsky et al., 2001) and similar conceptualizations such as nonlinear interdependencies (Schiff et al., 1996), as well as mutual information, coherence, phase-locking approaches (wavelet and Hilbert transform), and linear analysis methods (Netoff et al., 2004), are commonly used (Quiroga et al., 
2002). These methodologies have been applied to EEG recordings during epileptiform activity and have raised questions as to the presumed high synchronization preceding the seizure (Mormann et al., 2000; Chavez et al., 2003). In vitro studies have also cast doubts on this concept (Netoff and Schiff, 2002). Nevertheless, the recording of high-amplitude waveforms during seizures clearly indicates there must be highly synchronized activity somewhere in the brain. Typical scalp EEG recordings represent field potentials that include cellular spiking activity as well as synaptic potentials, and thus we can postulate that at least localized synchrony may be higher during seizures.

We wanted to undertake an in-depth analysis of interictal and ictal phase-locking patterns, focusing on magnetoencephalographic (MEG) recordings of generalized seizures in patients with different epileptic syndromes using the Hilbert transform approach. We observed higher local (in small neighboring cortical areas; see Results) synchrony in the recordings from the patients during the seizures; however, distant synchrony did not show a similar increase during most of the seizures and sometimes was even lower than during interictal states. Different frequency bands showed specific phase-locking patterns. It was also observed that, in the same patient, for specific channels, seizures can have a different time-frequency structure. These observations indicate that the concept of hypersynchronous activity during seizures may not be as accurate as previously thought.

\section{Materials and Methods}

$M E G$ recordings and selection of subjects. Four patients were identified for study on the basis of a high likelihood for seizure occurrence during the MEG recording. All gave informed consent to have the MEG recording performed. Patient 1 is an 18 -year-old woman with primary generalized epilepsy and absence seizures who is taking a low dose of valproate antiepileptic medication monotherapy, and whose electroencephalograms in previous years had documented a very active generalized 3-4 Hz spike and wave abnormality, sensitive to hyperventilation. The other three patients suffered from intractable epilepsy, and previous video-EEG investigations had documented the occurrence of multiple daily generalized tonic motor seizures (primarily during sleep in patients 2 and 3; diurnal and nocturnal in patient 4 ). Patient 2 is a 42 -year-old woman with two areas of focal cortical dysplasia situated deep in the right frontal lobe, visible on brain magnetic resonance imaging (MRI), and is receiving antiepileptic polypharmacy (phenytoin, lamotrigine, valproate, and topiramate at the time of recording). Patient 3 is a 21 -year-old woman with developmental delay and secondary (symptomatic) generalized epilepsy and is taking phenytoin, lamotrigine, and clobazam. A previous partial anterior corpus callosotomy had been performed at another institution many years before without benefit. Patient 4 is a 38 -year-old woman with a partial anterior right hemispheric hemimegencephaly-like structural abnormality on brain MRI who is taking carbamazepine and topiramate. Six non-epileptic individuals on no medications (three men, three women; age range, 23-36 years) were studied as control subjects.

Subjects were tested supine inside the magnetically shielded room. MEG and simultaneous EEG data were recorded continuously $(625 \mathrm{~Hz}$ sampling rate; DC, $100 \mathrm{~Hz}$ bandpass; third-order spatial gradient noise cancellation) using a CTF Omega 151 channel whole-head system (CTF Systems, Port Coquitlam, British Columbia, Canada).

Phase synchronization analysis. For each patient and control subject, 16 2 min segments of recording were analyzed. Each of these segments was initially demeaned and bandpassed. We used a constrained least square finite impulse response (FIRCLS) filter (Rosenblum et al., 1996) with a bandpass of $\pm 2 \mathrm{~Hz}$ around a central frequency. Frequencies from 3 to 55 $\mathrm{Hz}$ were studied in each subject (central frequencies for the FIRCLS filter). On these series, the Hilbert transform was applied, and successive values of instantaneous phases were derived from the corresponding analytic signal. These phase series were then analyzed using sliding windows of $2.5 \mathrm{~s}$. Other window sizes were also tested with similar results regarding the most conspicuous features observed and described in this study. In each window, the analysis consisted of extracting the mean phase coherence statistic as described by Mormann et al. (2000). Additionally, we calculated a significance measure, synchrony index (S) (Le Van Quyen et al., 1998), as the difference between the original and the mean surrogate value, divided by the SD of the surrogate. A significance $S$ value of 3 , representing 3 SDs, indicates a significant difference between the real pair and the surrogate mean population for that pair at $p=$ 0.0025 . Surrogates were obtained following the same procedure and parameters used for the original series, but applying a time shift between each pair. In this way, we ensure that the series are not phase locked in general. The time shift was selected randomly from 1 per sampling frequency $(625 \mathrm{~Hz})$ to the highest possible allowed by our data. A total of 200 surrogates were obtained for each pair of channels. For each subject, an $S$ value was obtained for each possible pair and each window. The number of channels in each MEG recording was $\sim 146$, not necessarily fixed because in some traces certain channels were discarded because of the presence of artifacts.

We considered "local" synchrony measurements to be the average of S values between channels separated by $<4 \mathrm{~cm}$ and "distant" phase locking to be the average of $S$ values separated by $>4 \mathrm{~cm}$. Neighbor gradiometers (magnetic field sensors) are separated by a distance ranging from 3 to 4 $\mathrm{cm}$. When defining $4 \mathrm{~cm}$ for local synchrony, we make sure that at least one neighbor is included in the analysis for each channel.

\section{Results}

\section{Description of patient data}

Patient 1 had one generalized absence seizure, brought on by hyperventilation. There was no associated movement artifact. A second attempt to activate a seizure with hyperventilation in a later recording epoch was unsuccessful (and unaccompanied by any measurable change in synchronization). Patient 2 had two generalized tonic seizures recorded, both arising spontaneously. Review of the accompanying on-line video and the combined EEG and MEG recordings showed essentially no associated cranial movement artifact with the clinical events. Patient 3 had 12 generalized tonic seizures recorded, all arising spontaneously. Review of the combined EEG and MEG recordings did show some high-amplitude, low-frequency cranial movement artifact during the clinical events. Figure 1 shows examples of the apparently synchronous ictal onset patterns recorded simultaneously by scalp electroencephalogram and magnetoencephalogram in patients $1-3$. Patient 4 had no seizures during the MEG recording, and so in this case, only interictal background activity was available for analysis.

\section{Local and distant synchrony}

The first question we addressed was related to the extent of synchronization during ictal and interictal activity and whether this was dependent on the time scale (frequency band). Phase-locking values were determined at several frequency bands, from 3 to 55 $\mathrm{Hz}$ (details for the windows used are described in Materials and Methods). Figure 2 shows, for three patients and one control subject, the local phase locking across all frequencies, defined as the average of $S$ values in every pair of channels that are separated by $<4 \mathrm{~cm}$, and the distant phase locking across all frequencies, defined as the average of $S$ values separated by $>4 \mathrm{~cm}$ (as detailed in Materials and Methods).

The graphs in Figure 2 represent phase locking during epochs ( 2 min each) in which the patients had a seizure. The enhanced local synchronization is clear in all of the patients during the ictal events, in almost all frequency bands (left column). Distant (Global graphs) phase locking is most evident in the case of the primary generalized absence seizure, at all frequency bands, and not just the 3-4 Hz peak frequency of the ictal spike and wave 

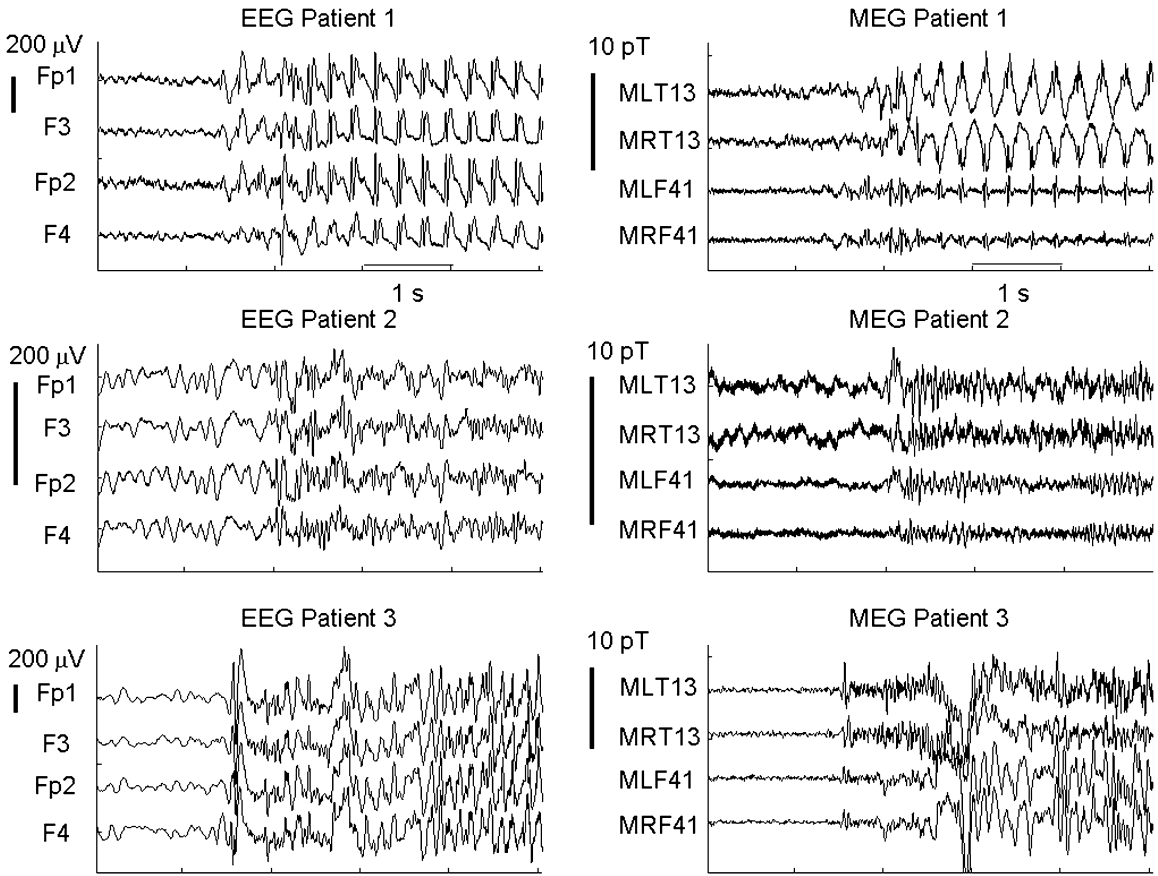

Figure 1. Ictal EEG (left) and MEG (right) onsets in patient 1 (top row), patient 2 (middle row), and patient 3 (bottom row). Left, The electroencephalogram shows widespread, apparently synchronous, generalized $3-4 \mathrm{~Hz}$ spike and wave (patient 1) or spike and wave followed by $20-30 \mathrm{~Hz}$ rhythmic $\beta$ ictal activity (patients 2 and 3). The top two EEG channels are the left frontal electrodes $\mathrm{Fp} 1$ and $\mathrm{F} 3$, and the bottom two EEG channels are the right frontal electrodes $\mathrm{Fp} 2$ and $\mathrm{F} 4$ in each patient. $\mathrm{Pz}$ was used as the common reference electrode. The cutoff frequency was $1 \mathrm{~Hz}$ for the high-pass filter and $100 \mathrm{~Hz}$ for the low-pass filter. Right, The top two MEG channels are the left and right anterior temporal regions, respectively, and the bottom two MEG channels are the left and right anterior frontal regions, respectively. The MEG trace shown in patient 3 was high-passed with a cutoff frequency of $6 \mathrm{~Hz}$ to partially eliminate the movement artifact that occurs $1 \mathrm{~s}$ after the onset of the seizure. This filter was applied only for visualization purposes and was not used in the numerical analyses of the study.

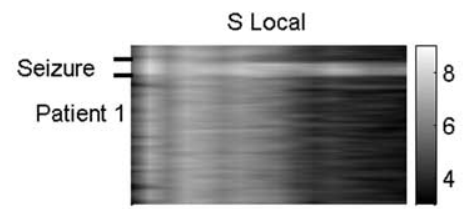

$\begin{array}{lllll}10 & 20 & 30 & 40 & 50\end{array}$

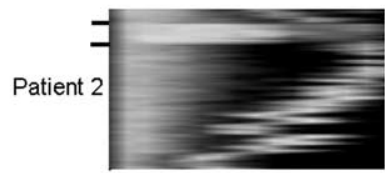

$\begin{array}{lllll}10 & 20 & 30 & 40 & 50\end{array}$

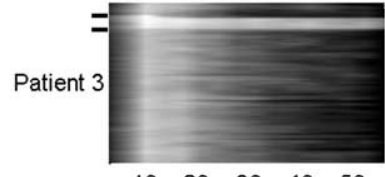

$\begin{array}{lllll}10 & 20 & 30 & 40 & 50\end{array}$

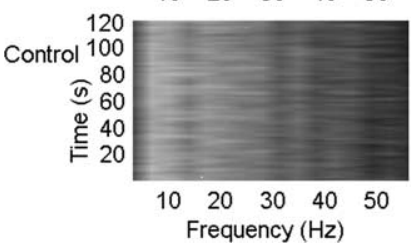

S Global

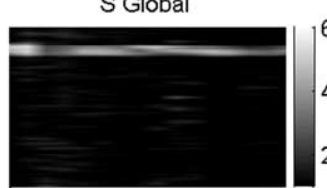

$\begin{array}{lllll}10 & 20 & 30 & 40 & 50\end{array}$

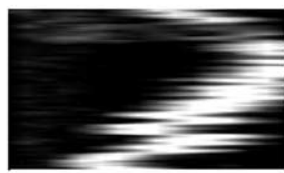

$\begin{array}{lllll}10 & 20 & 30 & 40 & 50\end{array}$

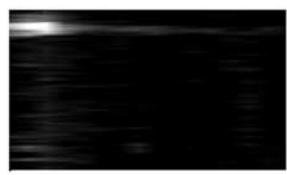

$\begin{array}{lllll}10 & 20 & 30 & 40 & 50\end{array}$

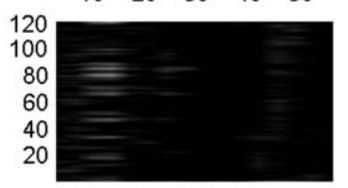

$\begin{array}{lllll}10 & 20 & 30 & 40 & 50\end{array}$
S Local and S Global at $36 \mathrm{~Hz}$ $\left.\right|^{61} 4$
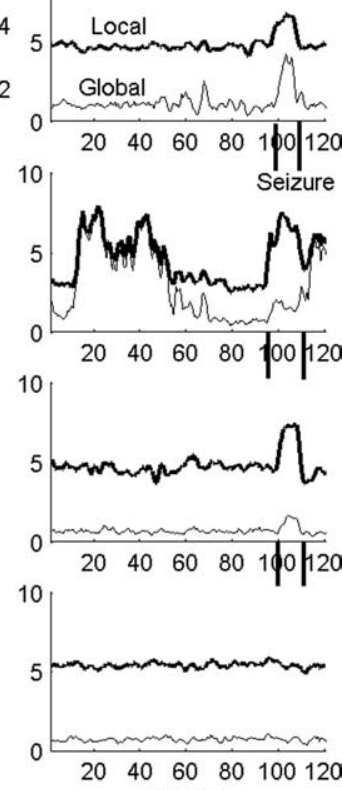

Figure 2. Distant versus local phase synchrony. Each row, from top to bottom, corresponds to patient 1, patient 2, patient 3, and control 1, respectively. First column (local synchrony), Mean $S$ value among channels separated by $<4 \mathrm{~cm}$. The onset and offset of each seizure are marked with thick lines. Second column (global, or distant, synchrony), Mean S value among channels separated by $>4 \mathrm{~cm}$. Third column, The thick line represents the local S values for the $36 \mathrm{~Hz}$ central frequency, and the thin line shows the global $S$ for the same frequency. activity (Fig. 1). Seizures in patient 3 did show some augmented distant synchrony in lower-frequency bands $(3-15 \mathrm{~Hz})$, although this may have been related in part to the low-frequency movement artifact visible during the clinical seizures in this patient. The progression toward the seizure in patient 2 merits some comments. Note the enhanced synchronization at different frequency bands as time progresses toward the seizure: just before the ictus, the phase locking is found at high frequencies $(>40 \mathrm{~Hz})$. Considering that the MEG signals represent a summation of intracellular currents in neurons receiving excitatory or inhibitory synaptic inputs, the phase-locking trend we see in this patient as the seizure approaches can be interpreted physiologically as starting with lowfrequency synaptic inputs (at the start of the episode; bottom part of the graph), which progressively recruit cells firing at higher frequencies that also result in higher frequencies of synaptic activity, so that at seizure onset most cells are probably firing at high frequencies. Thus, the observation of this "walk" of phase locking in the case of a secondarily generalized seizure could be the manifestation of the underlying cellular activities moving toward a recruitment of high-frequency firing cells. Perhaps related to this, it has been shown that frontal lobe onset seizures, when recorded intracranially, tend to start with high-frequency EEG activity (Fisher et al., 1992).

The plots of the time course of the $\mathrm{S}$ values (Fig. 2, right column) are useful to appreciate in detail the variations in local and global synchrony. Again we see here that only in the case of the absence seizure did both local and global indices have a similar increase during the ictus. For 36 $\mathrm{Hz}$, the relative jump in synchronization (relative to its baseline) during the seizure is higher in the global synchrony, although this is not necessarily the case for other frequencies. In patient 3 , with symptomatic generalized epilepsy, there is a lesser rise in global synchrony during the seizure, whereas in patient 2 , with a frontal onset secondarily generalized seizure, global synchrony increases only after the event. It can be seen that the global $S$ value is lower than the local $S$ value in all cases. No consistent trend in synchrony during the approach to seizure onset was detected across the patients. The time course of $S$ values in control subjects displayed only small fluctuations about the baseline, as depicted in the fourth graph in Figure 2. Note also that the baseline levels for local and global synchrony are very similar in the four cases. 
To examine in more detail the phaselocking patterns between specific cortical areas, we analyzed the synchrony between all channels, individually. The graphs in Figure 3 represent $S$ values in specific MEG channels (called "index channels" here) versus the rest of the channels on the scalp. We arranged the channels in each graph by order of proximity to the index channel. A finer determination of the synchrony pattern is achieved with this visualization. For patient 1 (top row), the channel selected as index was LF32; for patient 2 (middle row), the channel selected as index was LP12; and for patient 3 (bottom row), the channel selected as index was LP21. Each column shows a different frequency: four equidistant frequencies from 4 to $38 \mathrm{~Hz}$. During the absence seizure (patient 1), high synchrony values tended to be present even with distant channels at higher frequencies, but especially at the predominant $4 \mathrm{~Hz}$ time scale, whereas in the cases of tonic motor seizures, significantly high values are apparent only for neighboring channels. Note the cone-like appearance of the region of high synchrony during the seizure in patient 3, which is also evident in the third and fourth columns in patient 2 , indicative of a successive recruitment of neighboring areas into the phase-locked region, reaching the maximum distance in the middle of the seizure. This same mechanism appears to operate in reverse toward the end of the seizure, in this case with progressive desynchronization: more distant channels start to desynchronize first, followed by a progressive desynchronization of closer channels. This trend was seen for most of the channels. The cone-like structure (the cone is seen in three dimensions; in the two-dimensional plots shown in the figures this appears as a triangle) was seen in most areas, as depicted in Figure 4. Interestingly, this cone-like structure was not clearly evident at the lower-frequency bands in the case of the absence seizure (patient 1), which may be a manifestation of the presumed differences underlying seizure generation and maintenance in this epileptic syndrome. The thalamocortical reverberation characteristic of absences will probably cause a more uniform cortical recruitment because of the strong uniform thalamic input. We address this issue in the Discussion. The higher generalized synchronization observed in patient 3 at $4 \mathrm{~Hz}$ is attributable to the low-frequency movement artifact during the seizure (still visible in the traces shown in Fig. 1). Even under this circumstance, the well structured patterns are readily apparent at higher frequencies in which the movement artifact has no power. The bands of high synchrony present in patient 2 (26.6 and 38 $\mathrm{Hz}$ ) do not correspond to any visible artifact. Similar highsynchrony bands during interictal activity have been observed in previous studies [Le Van Quyen et al. (2003), their Fig. 4A].

In Figure 4, we explore the topographical distribution of the cone-like structure. Here, we select a window around one of the most well defined seizures in patient 3. Each plot is of the same kind as those shown in Figure 3, except for the short peri-ictal time span. Here, for each plot, the cranial position of the index
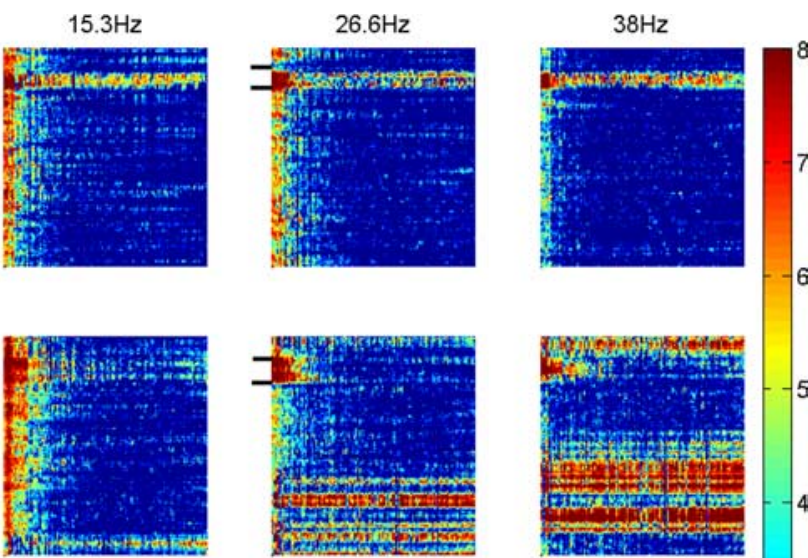

6
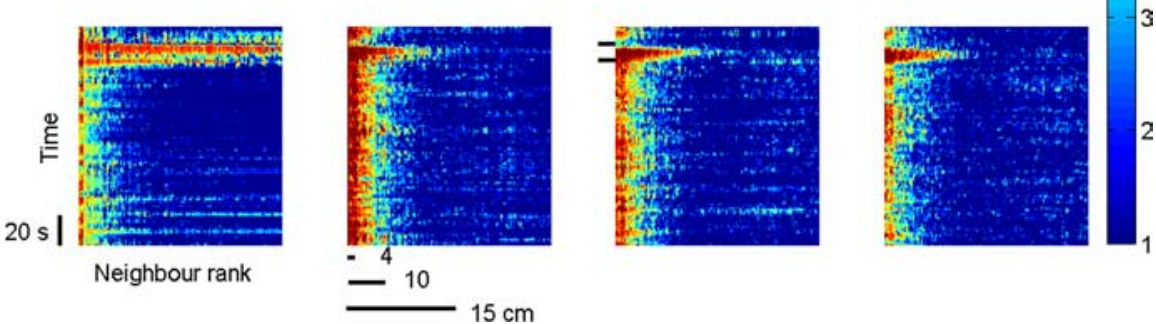

Figure 3. Plot of $S$ values for patient 1 (row 1), patient 2 (row 2), and patient 3 (row 3). A given channel is selected (index bottom indicate the approximate distance between the index and the channel assigned to that specific rank. The index channels Recordings obtained on other days without seizures in this patient did not show these prominent synchrony bands. The respective time courses of the raw EEG and MEG signals did not show any conspicuous difference during these bands of high synchronization.

channel is indicated on the diagrammatic head. Observe that the possible recruitment mechanism (indicated by the cone structure as mentioned above) seems to occur around the same area. The poor participation of more peripheral areas, especially in the frontal regions, could hypothetically be related to this patient's previous partial anterior corpus callosotomy, although this is speculative.

\section{Fluctuations in synchrony}

The phase-locking pattern between two specific channels was determined during the different ictal events in each patient to explore the possibility that synchronization during seizures may not be as fixed as commonly presumed. Although most of the channels showed relatively constant enhanced synchrony in a particular frequency band during seizures in the same patient, there were some that displayed highly variable synchronization from seizure to seizure, as depicted in Figure 5. In this figure, two channels were chosen in the patient with symptomatic generalized epilepsy, one in the left temporal region and another in the right frontal region, and five epochs ( $2 \mathrm{~min}$ each) are shown, containing seven seizures. The $\mathrm{S}$ value tends to be higher in most of the ictal episodes compared with the interictal periods, but note that there is one seizure (Fig. $5 d$ ) in which these regions did not synchronize; indeed they seem to show a lower synchrony during the ictus. There was a high variability of the synchrony pattern for each seizure, although they all belonged to the same patient. The frequencies in which higher phase locking occurs are in the range $6-30 \mathrm{~Hz}$ during the ictal events, with some excep- 


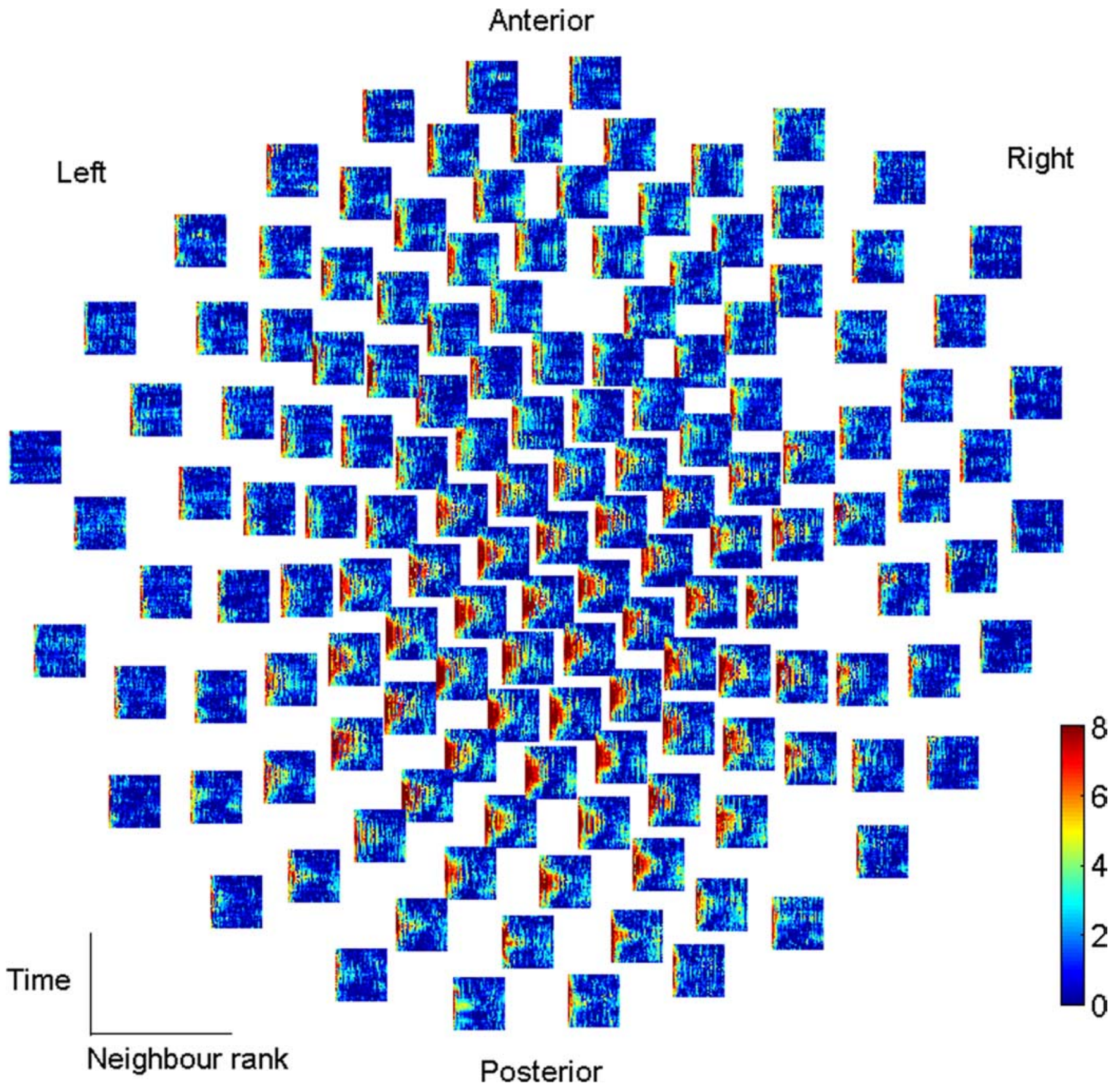

Figure 4. S plots for every channel during a seizure in patient 3 at $36 \mathrm{~Hz}$. Each square represents 5 values during the seizure shown in Figure 3 (bottom row) for each of the 146 channels. The position of each index channel is mapped on the diagrammatic head.

tions such as the seizure in Figure $5 e$, in which higher frequencies (up to $45 \mathrm{~Hz}$ ) show an increased significance value. To further illustrate that desynchronization between specific cortical areas also occurs in other syndromes, Figure $5 f$ shows one epoch in which the patient with absence epilepsy had two channels that had a reduced $S$ value during the ictal event compared with the preictal and postictal periods. However, although not uncommon, this marked desynchronization between particular channels during the ictal period was not the rule in the patients studied.

Comparison of synchronization patterns between control subjects and epileptic patients

One might conceive that during interictal periods the epileptic brain may display a higher degree of synchronization than the non-epileptic brain, both in anatomical extent and in magnitude, and therefore be prone to ictal synchronization or hypersynchronization. To address this hypothesis, we examined the resting synchronization patterns in six non-epileptic subjects and compared the data with that observed in the patients under study with epilepsy. Determination of the average extent of phase synchronization in these subjects revealed no significant differences when compared with the interictal recordings of the four patients. This similarity in the "basal" activity can be seen in Figure 2, especially in the right column. The similarity in the synchronization patterns between controls and patients is important because it argues against effects of the anti-epileptic medications on the synchrony we measured. Although these drugs are often effective at reducing seizures, their anti-seizure actions may not be measur- 

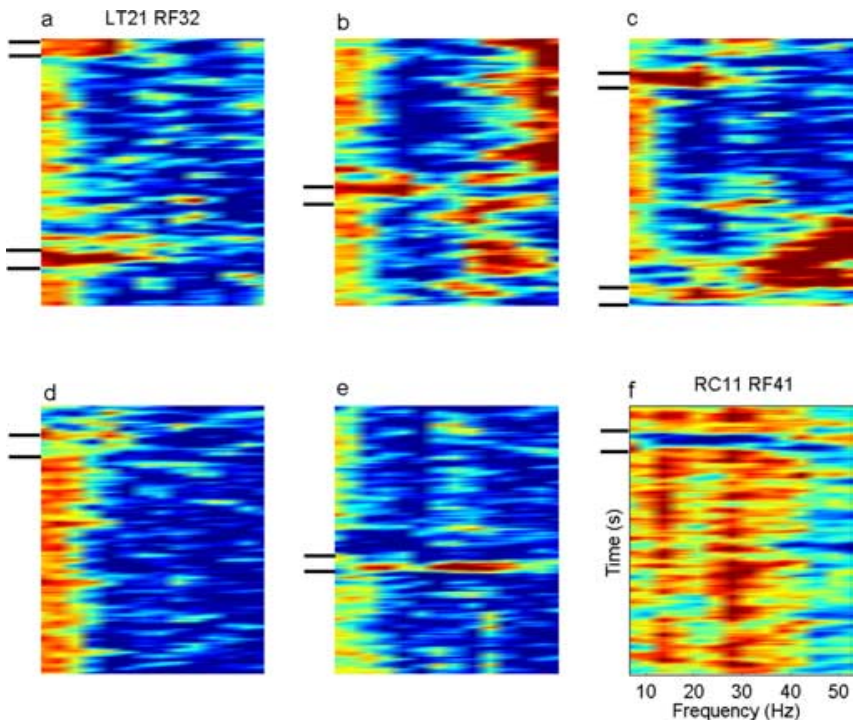

Figure 5. Time-frequency plots of fluctuations in ictal synchrony patterns. $\boldsymbol{a}-\boldsymbol{e}, \mathbf{S}$ values for two selected channels (LT21, RF32) in patient 3. Each figure corresponds to a 2 min segment. The onset and offset of each seizure are marked with thick lines. $\boldsymbol{f}, \boldsymbol{S}$ values for two selected channels (RC11, RF41) in patient 1 (absence seizure).

able by this type of analysis, and thus the drugs are not a confounding factor, at least in our study.

\section{Discussion}

Our observations suggest that epileptic paroxysmal discharges recruit neighboring brain areas into a somewhat fluctuating phase-locked state, whereas for distant regions the synchronization does not increase with the same absolute magnitude as in neighboring areas. Because the extent of synchronization in nonepileptic subjects was similar to that observed in the patients during interictal periods, we cannot conclude that neuronal networks of the epileptic brain show higher baseline synchronization than the non-epileptic brain.

Estimation of phase differences based on the analytic signal concept (Gabor, 1946) is widely used in neuroscience (Stam et al., 2003). Although estimation of coherence is also being used extensively to assess synchrony, coherence analysis does not distinguish between phase and amplitude dynamics and, in general, is not equivalent to phase synchronization (Tass et al., 1998, 2003). Thus, caution should be exercised when comparing results derived from different analysis techniques and recording methods. We must also consider that we are assuming that our measures of synchronization accurately represent physiological adjustments of relationships between characteristic times or phases (or frequencies) between interacting systems, despite the noisy nature of the MEG/EEG signals.

The observation of strong local synchrony agrees with a previous study using scalp EEG recordings, which reported that the measurement of an enhanced nearest neighbor phase synchronization during seizures was efficient in detecting seizure activity (van Putten, 2003). Indeed, the fact that large-amplitude field potentials are recorded during seizures is a demonstration of local synchronization with small phase difference. However, the physiological interpretation of synchronization as measured from these field potential-like (EEG/MEG) recordings is not trivial. In the case of the magnetoencephalogram, we are recording the summation of local field potential activity that is presumably producing sufficiently coherent currents in a number of cells to generate externally detectable magnetic fields. It should be noted also that, in contrast to the electroencephalogram, which detects primarily activity in the underlying cortical areas dominated by radially oriented extracellular currents, MEG recordings will be primarily sensitive to activity in the tangentially oriented cortex (sulci) that will produce dipolar patterns of magnetic field activity at the scalp surface on either side of the underlying sources. Thus, more superficial cortical sources will produce ingoing and outgoing magnetic fields at closely spaced MEG sensors compared with deeper sources that would produce more widely distributed field patterns. Local field potentials represent a summation of synaptic inputs to the area where the electrode is located as well as spike firing. Hence, synchronization in, for example, lowfrequency bands may be indicative of a common incoming synaptic drive, because most paroxysmal cell firing tends to be at higher frequencies. Thus, it is important to consider different time scales in neuronal synchronization (Netoff and Schiff, 2002), because there could be desynchronization in the cell firing (at short time scales) and synchronization at the slower time scale of bursting activity and synaptic inputs. Still, if there are strong synaptic inputs generating measurable, large-field potentials, this indicates that there is another brain area(s) that is synchronized and sending strong correlated input. These are indications of highly synchronized activity, but the origins may not be easily interpretable. Concepts such as functional connectivity (Aertsen and Preissl, 1990) have been developed to address the issue of interacting brain areas, which may provide a firmer basis on which to interpret observations related to synchronization.

Synchronization patterns in epileptiform activity have been studied for many years. The previous studies of Petsche et al. (1984) on penicillin-evoked seizures in rabbit cortex revealed that seizures begin locally and that the coherence in oscillation frequency is inversely proportional to the area of cortical tissue involved. More recent studies have described a decrease in synchronization during the preictal period (Mormann et al., 2000; Chavez et al., 2003), whereas others have found an enhancement in local synchrony (van Putten 2003). However, there were differences between the studies in the frequency bands at which decreases in preictal synchronization were found. In our study, we could not appreciate any reproducible trend in the changes in preictal synchronization at any particular frequency band studied (up to $55 \mathrm{~Hz}$ ). Although we used similar methods to detect phase synchrony, the other studies aforementioned were based on EEG recordings using subdural grids or intracortical depth electrodes. These methodologies have the disadvantage of needing a reference electrode, with consequent changes in phase synchrony that can be attributed to the reference (Fein et al., 1988; Zaveri et al., 2000). We have shown recently (Guevara et al., 2005) that estimation of phase locking using the Hilbert (or wavelet) transform, in the case of recordings with a common reference, can result in spurious synchrony and in particular that the amplitudes cannot be separated from phases, a precept of the analytic signal concept (Gabor, 1946). In vitro studies, in which action potential recordings from individual neurons are performed, are better able to explore in detail the nature of neuronal synchrony. In this regard, a recent in vitro study demonstrated desynchronization at fast time scales during seizure-like events and also failed to detect a pre-seizure period in terms of changes in synchronization (Netoff and Schiff, 2002), observations that agree with ours. Although one should use caution in comparing in vitro with in vivo data, these in vitro observations stress the importance of considering synchronization across several frequency bands.

We observed differences in the dynamics of the global syn- 
chronization patterns of seizures in the patient with absence seizures (patient 1) compared with the others, despite the widespread and generalized visual appearance of the ictal MEG and EEG recordings. The absence event in the patient with primary generalized epilepsy displayed the most extended global synchrony. This may conceivably relate to the accepted conceptualization that absence seizures rely on a "reverberating" thalamocortical loop for their generation and maintenance (Gloor and Fariello, 1988; Crunelli and Leresche, 2002). Hence, the strong low-frequency thalamic input will be equally "seen" by most cortical areas at the same time. A lesser increase in global synchrony was seen during seizures in the patient with symptomatic generalized epilepsy, in which a thalamocortical pathophysiological component is also presumed, albeit of a less organized and multifocal cortical distribution. For the patient with the focal (frontal) epileptic syndrome, global synchrony did not increase during the generalized seizure, notwithstanding the appearance of the MEG and EEG recordings. It would appear that seizures that are more strictly cortical in origin will have a more pronounced spread through the neocortex, resulting in distinct patterns of phase locking at several time points in different cortical areas. The observation that phase synchrony in lower frequencies is more distant than higher frequencies can be understood if we consider the nature of the recordings: higher frequencies $(>25 \mathrm{~Hz})$ could represent the intense spike firing during the seizure that may be harder to synchronize between distant areas, whereas lower frequencies represent bursting and synaptic input patterns that may be widespread during ictal activity throughout large areas.

We can question whether it is reasonable to expect phase synchronization in electrical brain recordings or whether this is a spurious outcome of the analysis method. The latter seems unlikely because phase synchronization occurs not only in periodic oscillators but also in chaotic or stochastic ones (Rosenblum et al., 1996). In general, synchronization is a generic property widely found in many systems, and in the case of the brain, we propose that the framework of periodic and aperiodic forcing (Hoppenstead and Izhikevich, 1997) could aid in comprehending neuronal dynamics. The current developments in the theory of stochastic synchronization (Pecora et al., 1997; Anishchenko et al., 2002) promise advances in the characterization and functional understanding of brain synchrony patterns.

It seems reasonable to expect that neocortical tissue will never be fully synchronized, even during paroxysmal activity representing the highly correlated firing of multitudes of neurons. There are theoretical considerations that suggest that the neocortical networks should not completely synchronize, and proposals have been made to consider the cortex as composed of relatively independent cell ensembles (Hoppensteadt and Izhikevich, 1998). Other theoretical studies of synchronized oscillations in the presence of noise have revealed that synchrony still occurs, but for a limited time interval (Stratonovich, 1967). Also, the proposed "small world" models of the neocortex (Sporns and Zwi, 2004), based on anatomical and functional observations, favor the idea of local rather than extended global synchronization. An important aspect to be emphasized is that of fluctuations in phase synchrony, apparent not only in our studies but also during normal, cognitive periods (Lutz et al., 2002; Gong et al., 2003). However, the fluctuations in phase synchrony between two specific cortical areas, from seizure to seizure in the same patient, are somewhat surprising considering the robust correlated firing and the recruitment of adjacent areas during ictal events. Within current theoretical frameworks of brain function that propose information processing to emerge from transient dynamic interactions in distributed brain areas (Bressler and Kelso, 2001; Friston, 2001; Varela et al., 2001), seizures can be considered abnormal extensions, in magnitude and in anatomy, of the normal transient synchrony patterns.

The observation of dynamical changes in synchronization patterns provides support for the notion of the continuous, nonstationary nature of brain activity and the fast transitions between coordinated activity states (Kryukov et al., 1990; Lopes da Silva et al., 1994, 2003; Friston, 2001; Perez Velazquez et al., 2003; Perez Velazquez and Wennberg, 2004). Indeed, study of the nonstationarities may reveal additional insights into seizure dynamics (Dikanev et al., 2005), and some methods can be applied that are able to handle nonstationarities, such as the search for unstable periodic orbits (So et al., 1998). Alterations in synchrony have been proposed to reflect relationships between neuronal activity in possible epileptic foci and surrounding areas (Chavez et al., 2003; Mormann et al., 2000). We propose an extension of this idea, in that the epileptic "focus" may be defined locally for each cortical area: any neuronal network that starts to synchronize with the neighbors can be considered a focus, with activity relationships that will vary between adjacent regions depending on the networks entrained and the frequencies at which they synchronize. Thus, the end result will be a local synchronization and distant desynchronization.

An interesting question from the theoretical perspective is whether the synchronization is stable or not, because this provides insight into the generation and dissolution of seizures. Considering the brief existence of most seizures (excepting the special cases of status epilepticus in which seizures do not stop spontaneously), and the difficulty at halting them even with strong external perturbations (Khosravani et al., 2003), we can propose that the synchronization manifold where the activity lies is resistant to perturbations (Hirsch et al., 1977; Josic, 1998; Kocarev et al., 2000) and thus requires profound changes in parameters if it is to be destroyed. In this regard, many parameters are dynamically changing during seizures, from inhibitory transmission (Perez Velazquez and Carlen, 1999) to the transient depletion of neurotransmitters because of the intense neuronal firing. Future, more refined studies should address the specific anatomical location of the measured synchronization and the possible functional role of synchrony fluctuations as the ictus advances, using novel methodologies proposed recently (Tass et al., 2003).

\section{References}

Aertsen A, Preissl H (1990) Dynamics of activity and connectivity in physiological neuronal networks. In: Nonlinear dynamics and neuronal networks, VCH (Schuster HG, ed), pp 281-301.

Anishchenko VS, Astakhov VV, Neiman AB, Vadivasova TE, SchimanskyGeier L (2002) Nonlinear dynamics of chaotic and stochastic systems. Berlin: Springer.

Bressler SL, Kelso JAS (2001) Cortical coordination dynamics and cognition. Trends Cogn Sci 5:26-36.

Chavez M, Le Van Quyen M, Navarro V, Baulac M, Martinerie J (2003) Spatio-temporal dynamics prior to neocortical seizures: amplitude versus phase couplings. IEEE Trans Biomed Eng 50:571-583.

Crunelli V, Leresche N (2002) Childhood absence epilepsy: genes, channels, neurons, and networks. Nat Rev Neurosci 3:371-382.

Dikanev T, Smirnov D, Wennberg R, Perez Velazquez JL, Bezruchko B (2005) EEG nonstationarity during intracranially recorded seizures: statistical and dynamical analysis. Clin Neurophysiol 116:1796-1807.

Fein G, Raz J, Brown FF, Merrin EL (1988) Common reference coherence data are confounded by power and phase effects. Electroencephalogr Clin Neurophysiol 69:581-584.

Fisher RS, Webber WR, Lesser RP, Arroyo S, Uematsu S (1992) Highfrequency EEG activity at the start of seizures. J Clin Neurophysiol 9:441-448. 
Friston KJ (2001) Brain function, nonlinear coupling, and neuronal transients. The Neuroscientist 7:406-418.

Gabor D (1946) Theory of communication. Proc IEEE 93:429-457.

Gloor P, Fariello RG (1988) Generalized epilepsy: some of its cellular mechanisms differ from those of focal epilepsy. Trends Neurosci 11:63-68.

Gong P, Nikolaev A, van Leeuwen C (2003) Scale-invariant fluctuations of dynamical synchronization in human brain electrical activity. Neurosci Lett 336:33-36.

Guevara R, Pérez Velazquez JL, Nenadovic V, Wennberg R, Senjanovic G, García Dominguez L (2005) Phase synchronization measurements using electroencephalographic recordings: what can we really say about neuronal synchrony? Neuroinformatics, in press.

Hirsch MW, Pugh CC, Shub M (1977) Invariant manifolds. New York: Springer.

Hoppensteadt FC, Izhikevich EM (1997) Weakly connected neural networks, applied mathematical sciences, Vol 126. New York: Springer.

Hoppensteadt FC, Izhikevich EM (1998) Thalamo-cortical interactions modeled by weakly connected oscillators: could brain use FM radio principles? BioSystems 48:85-94.

Josic K (1998) Invariant manifolds and synchronization of coupled dynamical systems. Phys Rev Lett 80:3053-3056.

Khosravani H, Carlen PL, Perez Velazquez JL (2003) The control of seizurelike activity in the rat hippocampal slice. Biophys J 84:1-9.

Kocarev L, Parlitz U, Brown R (2000) Robust synchronization of chaotic systems. Physiol Rev [E] 61:3716-3720.

Kryukov VI, Borisyuk GN, Borisyuk RM, Kirillov AB, Kovalenko YI (1990) Metastable and unstable states in the brain. In: Stochastic cellular systems: ergodicity, memory, morphogenesis (Dobrushin RL, Kryukov VI, Toom AL, eds), pp 225-358. Manchester, UK: Manchester UP.

Le Van Quyen M, Adam C, Baulac M, Martinieri J, Varela FJ (1998) Nonlinear interdependencies of EEG signals in human intracranially recorded temporal lobe seizures. Brain Res 792:24-40.

Le Van Quyen M, Navarro V, Martinieri J, Baulac M, Varela FJ (2003) Toward a neurodynamical understanding of ictogenesis. Epilepsia 44 [Suppl 12]:30-43.

Lopes da Silva FH, Pijn JP, Wadman WJ (1994) Dynamics of local neuronal networks: control parameters and state bifurcations in epileptogenesis. Prog Brain Res 102:359-370.

Lopes da Silva FH, Blanes W, Kalitzin SN, Parra J, Suffczynski P, Velis DN (2003) Dynamical diseases of brain systems: different routes to epileptic seizures. IEEE Trans Biomed Eng 50:540-548.

Lutz A, Lachaux JP, Martinerie J, Varela FJ (2002) Guiding the study of brain dynamics by using first-person data: synchrony patterns correlate with ongoing conscious states during a simple visual task. Proc Natl Acad Sci USA 99:1586-1591.

Mormann F, Lehnertz K, David P, Elger CE (2000) Mean phase coherence as a measure for phase synchronization and its application to the EEG of epilepsy patients. Physica D 144:358-369.

Netoff TI, Schiff SJ (2002) Decreased neuronal synchronization during experimental seizures. J Neurosci 22:7297-7307.

Netoff TI, Pecora LM, Schiff SJ (2004) Analytical coupling detection in the presence of noise and nonlinearity. Physiol Rev [E] 69:017201.
Pecora LM, Carroll TL, Johnson GA, Mar DJ, Heagy JF (1997) Fundamentals of synchronization in chaotic systems, concepts and applications. Chaos 7:520-543.

Perez Velazquez JL, Carlen PL (1999) Synchronization of GABAergic interneuronal networks in seizure-like activity in the rat horizontal hippocampal slice. Eur J Neurosci 11:4110-4118.

Perez Velazquez JL, Wennberg R (2004) Metastability of brain states and the many routes to seizures: numerous causes, same result. In: Recent research developments in biophysics, Vol 3 (Pandalai SG, ed), pp 25-59. Kerala, India: Transworld Research Network.

Perez Velazquez JL, Cortez MA, Snead III OC, Wennberg R (2003) Dynamical regimes underlying epileptiform events: role of instabilities and bifurcations in brain activity. Physica D 186:205-220.

Petsche H, Pockberger H, Rappelsberger P (1984) On the search for the sources of the EEG. Neuroscience 11:1-27.

Pikovsky AS, Rosenblum MG, Kurths J (2001) Synchronization. A universal concept in nonlinear sciences. Cambridge, UK: Cambridge UP.

Quiroga RQ, Kraskov A, Kreuz T, Grassberger P (2002) Performance of different synchronization measures in real data: a case study on electroencephalographic signals. Physiol Rev [E] 65:041903.

Rosenblum MG, Pikovsky AS, Kurths J (1996) Phase synchronization of chaotic oscillators. Physiol Rev Lett 76:1804-1807.

Schiff SJ, So P, Chang T, Burke RE, Sauer T (1996) Detecting dynamical interdependence and generalized synchrony through mutual prediction in a neural ensemble. Physiol Rev [E] 54:6708-6724.

So P, Francis JT, Netoff TI, Gluckman BJ, Schiff SJ (1998) Periodic orbits: a new language for neuronal dynamics. Biophys J 74:2776-2785.

Sporns O, Zwi JD (2004) The small world of the cerebral cortex. Neuroinformatics 2:145-162.

Stam CJ, Breakspear M, van Cappellen, van Walsum AM, van Dijk BW (2003) Nonlinear synchronization in EEG and whole-head MEG recordings of healthy subjects. Hum Brain Mapp 19:63-78.

Stratonovich RL (1967) Selected topics in the theory of random noise, Vol 1 and 2. New York: Gordon and Breach.

Tass P, Wienbruch C, Weule J, Kurths J, Pikovsky A, Volkmann J, Schnitzler A, Freund H-J (1998) Detection of $n: m$ phase locking from noisy data: application to magnetoencephalography. Physiol Rev Lett 81:3291-3294.

Tass PA, Fieseler T, Dammers J, Dolan K, Morosan P, Majtanik M, Boers F, Muren A, Zilles K, Fink GR (2003) Synchronization tomography: a method for three-dimensional localization of phase synchronized neuronal populations in the human brain using magnetoencephalography. Physiol Rev Lett 90:88101.

van Putten MJ (2003) Nearest neighbor phase synchronization as a measure to detect seizure activity from scalp EEG recordings. J Clin Neurophysiol 20:320-325.

Varela FJ, Lachaux JP, Rodriguez E, Martinieri J (2001) The brainweb: phase synchronization and large-scale integration. Nat Rev Neurosci 2:229239.

Zaveri HP, Duckrow RB, Spencer SS (2000) The effect of a scalp reference signal on coherence measurements of intracranial electroencephalograms. Clin Neurophysiol 111:1293-1299. 